\title{
In Search of a Cure for a Psychosis in Information Systems Design: Co-created Design and Metaphorical Appreciation
}

\author{
Leslie J. Waguespack ${ }^{1}$, Jeffry S. Babb ${ }^{2} \&$ David J. Yates ${ }^{1}$ \\ ${ }^{1}$ Bentley University \\ Waltham, Massachusetts, USA \\ lwaguespack@bentley.edu \\ jbabb@wtamu.edu \\ ${ }^{2}$ West Texas A\&M University \\ Canyon, Texas, USA \\ dyates@bentley.edu
}

\begin{abstract}
We postulate that a disconnect between stakeholders and designers, often rooted in an understandable preoccupation with technical rationality, limits how design research is conceptualized in the design science research community. We posit co-creation as a way to overcome this limitation that engages reflective design practice fostering a shared understanding of value among the designers/developers, users, analysts and others. Thus, co-creation is an essential ingredient for design satisfaction in many design endeavors. We proffer a theoretical foundation for envisioning design success as an artefact that realizes co-created conceptual metaphors compositing the objective and subjective qualities shaping the stakeholders' appreciative systems. This paper positions and advocates for a critical perspective on designer transcendence where design choices and actions are centered on a shared, but evolving, composite understanding of value and quality - satisfaction. Successful co-creative design emancipates users from concern for unnecessary technically rational aspects of artefact design. Further we propose a framework, grounded in semiotics, to hone and revitalize designer transcendence with a design emphasis on efficient and ideally frictionless interfaces - conceptual metaphors - to reduce asymmetry among stakeholder concerns.
\end{abstract}

\section{Introduction}

Overall, information systems development (ISD) and design provides information systems artefacts that serve the intensions of a stakeholder community. As a thought experiment, imagine the work required to perform a cognitive task that satisfies a human intension as a distance to be traversed. That distance is fixed in the moment by how the task is defined. Further, consider that this distance must be traversed by the sum of the work performed by both a computing apparatus and human agents. With no computing apparatus, all cognitive work must be accomplished by the human agents. As such, if the task is "comprehensible" to a capable computing apparatus, then the human need only signal the computing apparatus to accomplish the task. Further, when the distance traversed by the computing apparatus meets the human agent, then that meeting point denotes the apparatus's degree of technological sophistication or a state of the art.

If this experiment strikes you prima facie as "cut and dry," then you likely have fallen victim to the psychosis of technical rationality! The experiment's description obscures three mutually confounding realities: 1) human intension is not solely objective, 2) human intension is only imprecisely expressible, and 3) the social context of human intension is an ever dynamic and open system. Any conception of information systems design ignoring or discounting these realities is misguided! We further elaborate on the psychosis below.

\subsection{Design is a Community Effort}

In information systems development (ISD), there are two principle agencies generalized as the designers and the stakeholders. We use "stakeholders" to collectively describe the agents who are the primary source of the expressed IS intensions and who will judge a designed information system as satisfactory, suitable, and perhaps even significantly pleasing. We will refer to the developers as "designers" and enfold the developers who we will assert must transcend their role as technicians to collaborate as full partners in creating artefacts that both resonate and thrive. On the surface, the designer's role appears to be the specification of the structural properties of a designed object [39]. However, in this paper, we will establish that the designer's role goes much deeper than this to include design aspects of the aesthetic. Thus, while these two parties engage the endeavor mutually, it is the stakeholders whose satisfaction is preeminent and the designers who must espouse stakeholder aspirations.

An ideal relationship between the designer/creators and stakeholders might be as partners in co-creation. Co-creation, a term and concept which was borne out the marketing literature and conceived as a response to the new and arising possibilities of an inter-connection world via the World Wide Web and the Internet [38], 
implies a partnership between designers and stakeholders that reduces the distance between them with respect to information asymmetry and defining value [26, 27, 45]. The foundational idea is "the joint creation of value by the company and the customer; allowing the customer to co-construct the service experience to suit their context" [38, p. 8]. Moreover, methodological paradigms for software and systems development such as Agile methods championed just such co-creation by emphasizing dialog, access, transparency, and ongoing negotiation for risk management via frequent iterations of the design/development cycle $[1,6,40,45]$. Co-creativity focuses on the communicative aspects of the partnership such that shared understanding and vision may be achieved [5, 17, 32]. Each party, the designer and the stakeholders, must principally overcome an inherent information asymmetry between them to pursue a coalescent understanding that characterizes a co-created product or service $[7,18,34,58]$. To conceptualize on design research, and paradigmatically, on design science research, without this dynamic in the forefront, is to reduce the degrees of freedom available to our understanding.

\subsection{Focusing on the Essence of Design: Diagnosing and Treating the Psychosis}

The psychosis in information systems design is often grounded in information asymmetry between the conceptual domains of the designer and stakeholders [2, $12,24,25,27]$. We propose to treat this psychosis by reevaluating the essence of design. Redirecting focus away from the artefact itself as the principal product of design and towards conceptual metaphor as the means to neutralize information asymmetry while also re-conceptualizing stakeholder satisfaction emergent from both subjective and objective aspects of design.

In this paper, we present a theoretical foundation [10, 56] for co-creation and a framework for understanding co-creation as a reflective design practice establishing conceptual metaphor as the core of the artefact. Thriving Systems Theory provides a taxonomy and vocabulary to address objective and subjective dimensions of design quality [53]. Stamper's semiotic ladder delineates the abstraction and de-abstraction that occurs during the cocreation of metaphors to bridge the asymmetry between designer and stakeholder world-views [49] - an approach congruent with a philosophy and epistemology of science grounded in Critical Theory [31]. We explore the imperative for designer transcendence beyond a technically-rational epistemology of practice to engage in a reflective design practice that is sympathetic to the stakeholders' world-view. We harness Vicker's and Checkland's concept of appreciative systems to describe generative dynamics governing the world-view within which both the designers and stakeholders act and adopt their respective natural attitude of everyday life [30]. Both designers and stakeholders are natives of distinct cultures that have central tendencies and norms [43].

\subsection{Critical Realism and the Co-Creative Designer}

From a critical philosophy, we posit that to undertake co-created design practice [41] is to take a critical realist perspective that is open to the real mechanisms that may underlie observed phenomena [15]. (See Figure 1.)

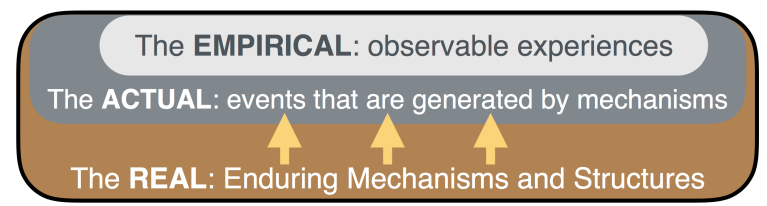

Figure 1. Critical Realism: Domains of the Real [31]

To extend design centered in technical rationality to design that is co-created requires a reflective design practice that considers the appreciative systems of stakeholders. Within such transcended design, the reflective designer relies on both empirics and interpretivism to question assumptions, to ponder governing variables, and to consider links between ontology and epistemology [43, 44].

In the critical realist view, the stakeholders' appreciative system and the structure and function of the system artefacts reflect one another; just as the ontology and epistemology of an underlying systems development methodology shape context and prefigure the developers' underlying conception of quality. For example, given their heritage, Agile methods have largely evolved in parallel with the ontology and epistemology of co-created design $[40,41,52]$. However, the ethos espoused in the Agile Manifesto does not entirely eschew the historical antecedents of software engineering and the epistemological assumptions embedded in the engineering metaphor. Thus, it is not surprising that with few exceptions, for example interaction design in Extreme Programming (XP) [1], technical rationality dominates the epistemology of Agile practice. As alluded to earlier, this may progress to a psychosis within the methodology as technical rationality alone is insufficient to realize the full benefit of Agile practice and the co-creation enshrined within that practice.

\subsection{Co-created Design and Design Science Research}

We examine co-created design as currently conceptualized in the design science research (DSR) literature and identify opportunities to better conceptualize co- 
creating within the DSR literature. Specifically, this paper is a knowledge contribution to the emerging area of co-created design in the context of a mature information system design process. (See Table 1.) Our framework for engaged and effective communication at different semiotic layers in co-created design represents an important means to achieve utility and fitness [9] in Hevner \& Drechsler's relevance and rigor cycles [6, 8, 13]. Closely related knowledge contributions -- also shown in Table 1 -- include the DSR work of Park \& Park [35], which focuses on reflexivity instead of metaphor, and the information systems design (ISD) co-evolution work of Vidgen \& Wang [52], which focuses on management and process more so than design and communication.

Table 1. Related Work in a DSR Knowledge Contribution Framework, adapted from [11]

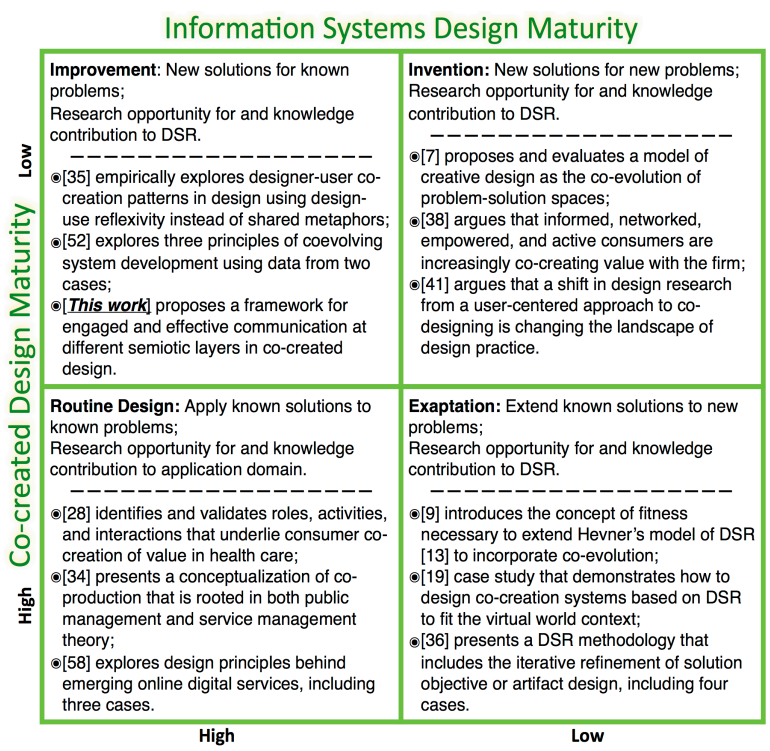

The paper proceeds as follows to elucidate both the psychosis of design and frame our search for a "cure." We examine the role of metaphor as a fundamental medium of communication between designer and stakeholder in synthesizing a shared, composite appreciative system to delineate satisfaction. We present the case for a marriage between technical rationality and appreciative systems as a necessary pre-requisite for co-created design. The penultimate section proffers our solution framework, grounded in semiotics, for both practitioners and researchers to consider. We conclude with a summary and opportunities to expand both the framework and its use.

\section{Knowing, Metaphor, the Praxis of Choice, and Appreciative Systems}

The human intellect contemplates existence in two fundamentally different contexts: a) in the world, and b) of the world. Iain McGilchrist attributes the divided locus of cognition to the right and left hemispheres of the human brain [29]. This division is the basis for understanding human memory, cognition, re-cognition, abstraction, tacit knowing, learning and imagination; all of which play essential roles in the agency of appreciative systems and metaphor - intrinsic to decision-making and design-as-a-verb. Successful design practice hinges on effectively communicating objective and subjective concepts. Metaphor is the hinge pin that conceptually integrates the objective and subjective. A synopsis of McGilchrist's account of neuropsychology explicates metaphor, appreciative systems, and praxis of choice.

\subsection{Lived Context vs. Abstracted Recollection}

The right hemisphere in the human brain takes in experience in context; all the coincident sensory input is processed as a whole. The left hemisphere does not; it re-forms experience digested into vignettes organized and encoded as property-laden abstractions. Each snippet of memory is characterized and categorized as conceptual metaphor stored for subsequent recall by means of attribution to context, sensory comparison, or analogical similitude. Where the right hemisphere indwells in the moment (in the world), the left hemisphere interprets the moment re-cognized, deciphered through an internalized world-view (of the world), a multidimensional network of associated metaphors.

The left hemisphere's cognition is calculating and deliberative; probing and testing for re-cognised abstraction. The left hemisphere realizes knowing in linguistic terms of vocabulary, syntax and semantics. Where the right hemisphere experiences the world viscerally, the left hemisphere's dependence on language predisposes an objectified world-view formed by hypothesis and proof - naturally sympathetic to a technical rationality. The right hemisphere's cognition is grounded in lived experience - being there in-the-moment. Unconstrained by language, its knowing is more tacit, intuitive; less deductive, as in "knowing more than you can tell" [37, p. 131]. The right hemisphere is the marvel of associative cognition, it emits response to stimulus reflexively, instinctively, intuitively through metaphorical recognition rather than calculation. The clinical evidence reveals that the right hemisphere thrives on framing and reframing conceptual spaces to (re-)interpret immediate experience. It fills in the gaps in language and "reads between the lines."

The right and left hemispheres interoperate in concert translating and pattern matching metaphor to the stream of awareness. As the right hemisphere scans, the left subconsciously sifts through vignettes that propose explanations for experience at hand. In effect, re-cognizing is first and foremost re-collection. Recognizing 
and conceptualizing are only possible in terms of what is already experienced.

\subsection{Language: Signs to Words to Metaphor to Knowledge to Culture to Value}

Fundamentally, knowledge and language engage the same currency, ideation. The earliest accumulation of "knowledge" consists largely of direct physical or sensory, experiential, objective "knowledge" stored without abstracting or encoding of language. Before the onset of language skills, this objective "knowledge" is operative as tacit knowing free of reflection or calculation, unselfconscious [37]. Language as basically a system of encodings, signs denoting concepts, evolves to enable the expression of conceptual metaphors. Metaphors emerge from the abstraction performed predominantly by the left hemisphere. Metaphorical expressions entail a displacement of physical experience to express abstract concepts metaphorically. The right hemisphere applies displacement of concepts to map experiences to metaphors [42]. Metaphor in linguistics is a poetic device. But in neuropsychology, metaphor is the device of ideation and abstraction in cognition [21, 53]. Cognitive psychologists account "knowledge" largely as a collection of conceptual metaphors. Learning is accumulating and refining theories of which metaphor is the intrinsic operative.

Processes of metaphor, or the displacement of concepts, are essential processes in the development of new theories, .... Each process of displacement revolves around the establishment of symbolic relations between the old theories and the new situation [42, p. xi].

Conceptual metaphors facilitate efficient communication by framing detail and naming intended (rather than literal) meanings. A system of conceptual metaphors is a cultural glue, normalizing vocabulary, values, and ceremonies in a social group's culture (e.g. coworkers, professionals, stakeholders) [20, 22]. Conceptual metaphors provide a trajectory for concept evolution by replacing, repurposing, or reinterpreting displaced concepts to satisfy a changing sociotechnical context [32].

The interdependent behavior of the left and right hemispheres reflects a praxis of choice combining technically rational and metaphorically subjective aspects of knowing. The psychological interplay of tacit, literal, and metaphorical knowledge argues that design quality must be grounded in a broader epistemological domain than technical rationality alone. Vickers' theory of appreciative systems expands that domain by attending explicitly to the dynamics of the sociotechnical context where the natural emergence of the concept of artefact quality unfolds in an ongoing cycle of reflection and adaptation $[17,25,33,51]$.

\subsection{Appreciative System: Situated Satisfaction}

The technical rationality that predominates in the natural sciences is an objective and positivist philosophy of decision making. It embraces the vision of an optimal outcome assured by a succession of choices pursuing a predetermined, calculable, and objective goal. Vickers' concluded after long governmental experience that, as an exclusive premise for decision-making in social contexts, it was fallacious [6]. Vickers' response was a decision-making epistemology emphasizing Weltanschauung [3, 16], a world-view authored and stewarded by stakeholder(s) summarized as follows:

1) a social context is a dynamic and open system; although it may be possible to isolate a snapshot of a world-view at a point in time, change in a social context is inevitable and perpetual,

2) judgements of fact may be objective, but judgements of value are always subjective - satisfaction is contingent on both,

3) unlike goal-seeking that promises an eventual terminus of optimality within an objectified ideal; an appreciative system strives for relationships among the facts and values that stakeholders apprehend as satisfying - while avoiding relationships that are not,

4) a choice should reflect judgements that honor both facts and value,

5) choices stem from judgements of the status quo; choices alter the status quo to influence subsequent judgements, and

6) satisfaction is served by a continuous cycle of judgement and choices attuned to the dynamic social context.

An appreciative system is a complex and emergent agency of choice in stakeholder behavior situated in a sociotechnical context [5]. Cultural assumptions, values, and attitudes converge to form the individual stakeholder's intellectual and emotional frame of reference.

The appreciative settings condition new experience but are modified by the new experience. Such circular relations Vickers takes to be the common facts of social life, but we fail to see this clearly, he argues, because of the concentration in our science-based culture on linear causal chains and on the notion of goal-seeking [4, p. 263].

Stakeholders possess appreciative systems individually as their experience and judgements are personal. But, appreciative systems also exist in community knowledge, norms, and aspirations held in common that characterize their culture: social, professional, religious, or intellectual - a shared world-view. Any human conception of satisfaction is founded upon an appreciative system subject in part to the subjective interpretation of their norms and aspirations - cultural metaphors. 


\subsection{Metaphor Unites Reason and Imagination}

Although the definition of rationality may be "the quality or state of being reasonable, based on facts or reason [57]," it is a mistake to assume that technical rationality reflects the sum of all reason.

Reason, at the very least, involves categorization, entailment, and inference. Imagination, in one of its many aspects, involves seeing one kind of thing in terms of another kind of thing - what we have called metaphorical thought. Metaphor is thus imaginative rationality [20, p. 193].

As there is no denying the shared agency of objective and metaphorical (subjective) modes of awareness of the world is at play in decision-making, it seems natural to acknowledge and leverage both modes in the practice of design.

Metaphor is one of our most important tools for trying to comprehend partially what cannot be comprehended totally: our feelings, aesthetic experiences, moral practices, and spiritual awareness. These endeavors of the imagination are not devoid of rationality; since they use metaphor, they employ an imaginative rationality [20, p. 193].

\section{Integrating Technical Rationality and Appreciative Systems}

An artefact exhibits four aspects of existence: $D e$ sign Intension, Ontology of Structure \& Function, Construction, and Stakeholder Experience. Design Intension anticipates what a human will comprehend of an encounter with the materialized artefact. That intension is prescriptive and predictive of a user's experience within a social context. The Design Intension designates, systematizes, and articulates the composition of the pertinent concerns of stakeholders: owner, investor, designer, builder, installer, manager, maintainer, user, end user or bystander. Ontology of Structure \& Function is the description of the stakeholders' obligations along with the commensurate artefact functionality (i.e. material structures and behaviors) at a stakeholder's bidding to satisfy those obligations. An apt metaphor for the $\mathrm{On}$ tology of Structure \& Function would be the tokens, moves, and rules of play for a board game where the players' decisions determine the course and outcome of play. In Simon's terms this is the specification of the "outer" environment of the artefact [46]. Construction is the result of materializing the intension through some process of expression or fabrication employing a medium of construction. In Simon's terms this is the realization of the "inner" environment of the artefact [46, p. 8]. Stakeholder Experience is what the stakeholders comprehend of their encounter with the constructed artefact that they sense as a degree of satisfaction. Once constructed, an artefact exists independent of its creators. It has a distinct identity with characteristics determined only by its construction. Various stakeholders will experience artefact characteristics differently, mediated personally through their individual world-view.

\subsection{Technical Rationality: Necessary for "Truth," Insufficient for "Satisfaction"}

What distinguishes an artefact from an object of nature is the human agency that imbues it. The conception and manipulation of an artefact's material characteristics adhere to the physical, technically rational world formed and governed by evidence subject to test and proof. Proven material facts reflect the laws of nature (as best we understand them).

An artefact exists situated in a social context that by its nature is an open system. As such, the whole of the artefact's environs form a design space of innumerable choices; the most numerous of which result from the subjective aspect of appreciative systems. Although both Schön and Simon dismiss the exclusivity of technical rationality's role in design decisions, they admit that "choosing among alternatives" permeates designas-a-verb regardless of the impossibility of enumerating all alternatives [47, p. 397]. Simon coined (and later Schön adopted) satisficing, as a workaround to technical rationality [46]. Satisficing proposes satisfaction rather than optimality as the goal of design. Satisficing relies upon constraining the domain of design choices to a closed system to facilitate an "adequate solution" search. Adequacy is justified by the solution's congruence both objectively and subjectively with an operative appreciative system. Tractability is achieved by constraining the range of design choices (aka reducing the design space). Adequacy and tractability are achieved with the Ontology of Structure and Function with satisfaction stipulated as a composite appreciative system negotiated among the stakeholders.

An Ontology of Structure \& Function identifies classes, individuals, relationships, and attributes (data and behavior) that depict the Design Intension ontologically within a relevant, social context. It defines and describes the metaphorical universe of functionality that stakeholders are able to exercise and experience in the artefact's operation. Developed in collaboration with the community of stakeholders, the Ontology of Structure \& Function describes information objects and transactions reflecting the operational obligations of all the stakeholders and the commensurate artefact behaviors prescribed to suffice those obligations. The expression of the Design Intension as an Ontology of Structure \& Function explicitly delineates a scope of operation and impact while providing a baseline to: 1) delineate the design space, 2) validate the domain of "adequate 
search," and, 3) establish the criteria for verifying the artefact's satisfactory operation as constructed. The coalescence of the design community's composite appreciative system (objective: primarily technically rational and subjective: attending to aesthetics and cultural norms) establishes artefact specific standards of quality with which to align the artefact's fabrication using the medium of construction.

The medium of construction is the substance, material, or components used to fabricate an artefact. While the intensional aspect of an artefact may be limited only by imagination, the materialized artefact is constrained by the medium of construction. The intrinsic characteristics of the medium of construction impact the stakeholder's experience if only by adding layers of syntax and intended (or unintended) nuance to the artefact's expression of intension. Ideally, the most efficient medium of construction is one where the translation of intension into artefact requires the minimum of either syntactic layering or unintended nuance. Although the Design Intension aspires to prescribe the Stakeholder Experience of the artefact, that actual experience is only achievable or observable in an actual, live encounter with the constructed artefact. Rendering the artefact is therefore intrinsic to design-as-a-verb.

\subsection{Naming and Framing Satisfaction}

The value of any artefact is the extent to which it enhances the stakeholders' sense of satisfaction in their social context. Thriving Systems Theory (TST) choice properties set out a taxonomy and vocabulary for naming and framing both objective and subjective dimensions of design quality. TST provides a framework to negotiate and articulate a community-shared appreciative system - their shared definition of "satisfactory" [53]. Where the stakeholders' intension aligns with their experience of the artefact, the artefact is instrumental to a living system, in harmony with and enriching their world-view, Weltanschauung [3, 4]. Where the artefact's Design Intension and Stakeholder Experience resonate in materializing an authentic expression of their values (their culture) - this is a signature quality of a thriving system. As such, the artefact not only expresses the intensions of the stakeholder, but it also enlightens, clarifies, and inspires in the personal interaction with the artefact. If an artefact is a system that thrives, it extends and enriches the stakeholder community's ability to achieve their tacit as well as conscious pursuit of satisfaction. This resonant satisfaction is metaphorically articulated as a sense of beauty (i.e. "a beautiful: architecture / building, theorem / formula, program / algorithm, computer system / application, painting / statue, etc."). When an artefact achieves resonant satisfaction, it is a thriving system, a thing of beauty [53]!

\section{Design-as-a-verb: Semiotic Convergence}

Situated in a social context is the natural circumstance of an artefact. Design is a quest for satisfaction in a dynamic of culture and ever-advancing technological opportunities, so to make sense of it we must adopt an experientialist paradigmatic perspective on design that is best examined through the lens of semiotics. Stamper presents a semiotic framework that explicates the expression and transmission of ideas, knowledge, and meaning through human communications [23, 48, 49]. The framework (aka the semiotic ladder depicted in Table 2) orients and categorizes design concerns spanning a sociological and technological landscape that artefact development must navigate in the pursuit of stakeholder satisfaction. The "ladder" characterizes layers of artefact abstraction. The layers represent a continuum of properties that inform an artefact's structure, behavior, and valuation to span the physical and social, material and conceptual in aligning the Stakeholder Experience with the Design Intension. Each layer intimates constructs as gradients that interpret the artefact metaphorically articulated in a vocabulary appropriate to that semiotic layer. Each layer molds the artefact depiction in relation to its context of semiotic concerns.

The semiotic ladder provides a framing analogous to the architecture of a multi-level virtual machine where the details of the supporting layers are encapsulated by each step to provide a homologous array of structural and behavioral appliances with which to express successive degrees of abstraction and interface. Unlike computer architecture however, the semiotic ladder extends beyond the physical, empirics, and syntactics to stratify psychological and sociological dimensions.

Table 2. The Semiotic Framework [23]

\begin{tabular}{|c|l|}
\hline $\begin{array}{c}\text { Semiotic } \\
\text { Ladder }\end{array}$ & \multicolumn{1}{|c|}{ Semiotic Layer Description } \\
\hline Social World & $\begin{array}{l}\text { Beliefs, expectations, functions, commitments, contracts, } \\
\text { law, culture }\end{array}$ \\
\hline \hline Pragmatics & Intensions, communications, conversations, negotiations \\
\hline \hline Semantics & $\begin{array}{l}\text { Meanings, propositions, validity, truth, signification, } \\
\text { denotations }\end{array}$ \\
\hline Syntactics & $\begin{array}{l}\text { Formal structure, language, logic, data, records, } \\
\text { deduction, software, files }\end{array}$ \\
\hline \hline Empirics & $\begin{array}{l}\text { Pattern, variety, noise, entropy, channel capacity, } \\
\text { redundancy, efficiency, codes }\end{array}$ \\
\hline \hline Physical & $\begin{array}{l}\text { Signals, traces, physical distinctions, hardware, } \\
\text { component density, speed, economics }\end{array}$ \\
\hline
\end{tabular}

In the practice of design, the semiotic ladder is itself a conceptual metaphor framing and naming the layers of abstraction that design reflection must attend to: (a) the objective quality of the artefact as an agent of computation (i.e. technical rationality: physical, empirics, syntactics) and (b) the subjective quality of the artefact's cultural resonance that satisfies stakeholders in an evolving social context (i.e. appreciative systems: semantics, pragmatics, social world). 
A product of any design process exhibits an intrinsic dualism - as both metaphor and artefact. (See Figure 2.) First, the product reflects a metaphorical agency in its design intentions:

- conceived (fundamentally) in a social world,

- translated through the pragmatics of culture, and

- expressing desired outcomes and qualities of performance.

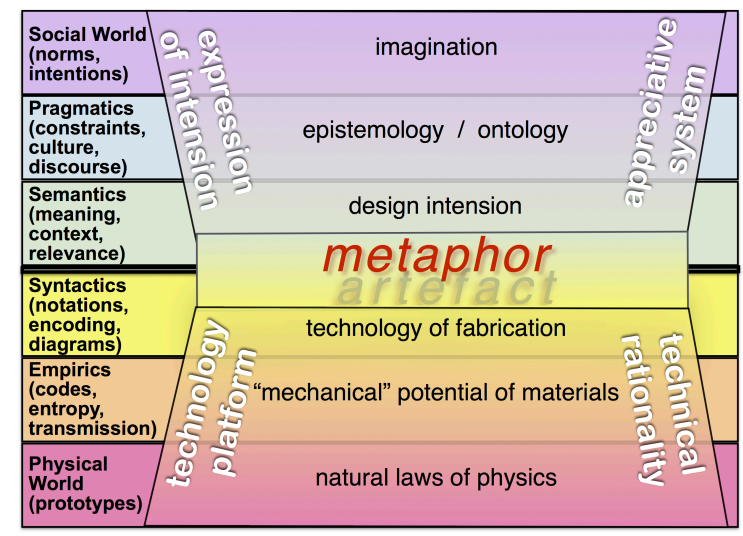

Figure 2. Marrying Technical Rationality and Appreciative Systems

Second, it demonstrates material artefact agency in its design intentions that reflect:

- conformance to the laws of nature,

- practicality in applying the medium of construction, and

- user experiences of outcomes and performance.

Figure 2 appears to orient the metaphor/artefact at a figurative median between the social world and the physical world. In fact, how design choices apportion the cognitive task load fulfilled by the artefact versus the load left to the faculties (ingenuity and effort) of the client/user will determine what orientation the designers and stakeholders perceive. Figure 3 demonstrates a variety of distinct placements of metaphor/artefact on the semiotic ladder due to design choices apportioning cognitive load differently - in this case, five depictions of the evolving design of long distance communication. The progressively greater distance from the baseline of materiality shows technology's evolving capacity to bear an increased cognitive load in the communication activity. A co-evolution in Figure 3 is notable between: a) the artefact's design in response to opportunities arising from advances in technology and b) the changing social conception of satisfactory long distance communication informed by a succession of artefact designs with increased capability. The position of the artefact's metaphorical conception (convergence of imagination and materiality) above the base of the ladder denotes the degree to which the designer has transcended a centeredness on technical physicality to advance toward the stakeholder's intension conceived in the social world; and the degree to which the artefact is able to emancipate the stakeholder from implementation details.

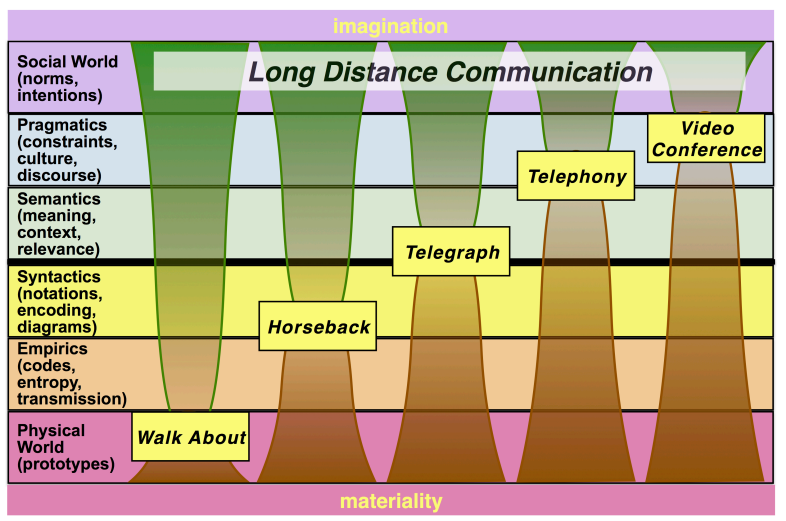

Figure 3. Examples Apportioning Cognitive Load

Figure 3 echoes Tesler's law of the conservation of complexity: the sum of complexity entailed in any artefact interaction is determined by that encountered by the user plus that subsumed by the artefact [50]. Reducing the complexity experienced by the user requires an equal, compensating increase of complexity in the technological sophistication of the artefact.

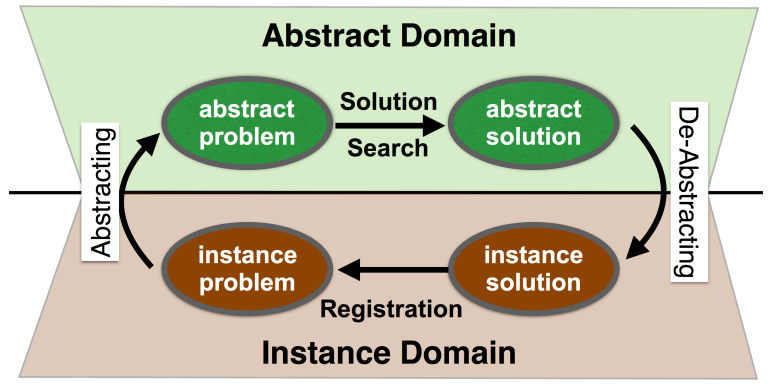

Figure 4. The Conceptual Interface between the Metaphor and the Constructed Artefact [22]

\subsection{The Juncture of Abstraction and Instance}

Figure 4 depicts the design theorizing framework necessary in developing theory $[10,56]$, a junction of abstract and instance domains. Although focused by its authors on design theory, it also explains the convergence of the imaginative and materiality aspects of design practice as framed by the semiotic ladder. The interplay of metaphorical and artefact agency in semiotic convergence echoes the iterative, cyclic process of problem/solution trials [7,36] across the abstract and instance domains of design theorizing. The theorizing threshold described in [22] corresponds to the metaphor orientation on the semiotic ladder reflecting the degree of designer transcendence. That transcendence results 
from a synthesis of technological skill for discerning design choices that absorb cognitive load and the designer's ability to internalize the Design Intension from the stakeholders' perspective.

In co-creative design, the theorizing threshold is a negotiated juncture of Design Intension and artefact realization. A unifying taxonomy and vocabulary of design quality enriches the negotiation process by organizing and normalizing the articulation of objective and subjective intensions [53].

\subsection{Marrying Technical Rationality and Appreciative Systems}

In the earliest experience of design, the stakeholder and the designer were one in the same; there was no "division of mind." The operative appreciative system and skill with the medium of construction were joined. The practice of design today must contend with the "divided mind" where what is known and what is valued must be "normalized" - encumbered by the challenge of communication among individual designers and stakeholders, a design community. Hence, we assert an unequivocal need for co-creative design.

Design is a search for a sociotechnical resonance wherein the stakeholders' experience an artefact in harmony with their shared appreciative system - where it is sustained and renewed through reflection dedicated to preserving that resonance. "Designing" cannot be simply a prelude to the delivered artefact, but it must be an ongoing process in the artefact's emergence - a cycle of reflection and renewal informed by the stakeholders' experience of the artefact in situ, the "live" social context $[9,55]$.

Historically, the focus of design has hinged largely on the nature of and skills with the medium of construction grounded traditionally in a technically rational perspective where design tasks are set as problems of logic or mathematics to be solved. But, the true problem that has emerged is how to set the problem, who sets it, and what are the terms for eventually assessing success. Those terms reside in the psychological and sociological dimensions of the design process [5, 17, 32].

In Figure 5 the designers' expertise (A) is traditionally grounded in their technically rational understanding of materiality - crafting edifices of technology that rise to meet the Design Intensions of the stakeholder community (B). The stakeholders' expertise (B) is naturally invested in the enterprise they pursue apart from the implementation intricacies of the medium of construction. Co-creative design interweaves the expertise of $(\mathrm{A})$ and the expertise of (B) to bridge their repertoires and appreciative systems through the dual agency of metaphor and artefact (C). This is the integration, the marriage, of technical rationality and appreciative systems.
Marriage is an apt metaphor for the fecund union of technical rationality and appreciative systems in a cocreative and emergent practice of design. Co-creation promotes collaboration of complementary points of view and encourages both designer and stakeholder to engage in mutual reflection that nurtures a genuinely shared conception of design quality in both product and process. Where satisficing evades the massively complex open system of the social context, integrating the aesthetic nature of the appreciative system civilizes an otherwise sterile conception of human agency. Aesthetics respects the tacit knowing ("wisdom") of living structures that designers and stakeholders accumulate through their experience in forming their respective repertoires.

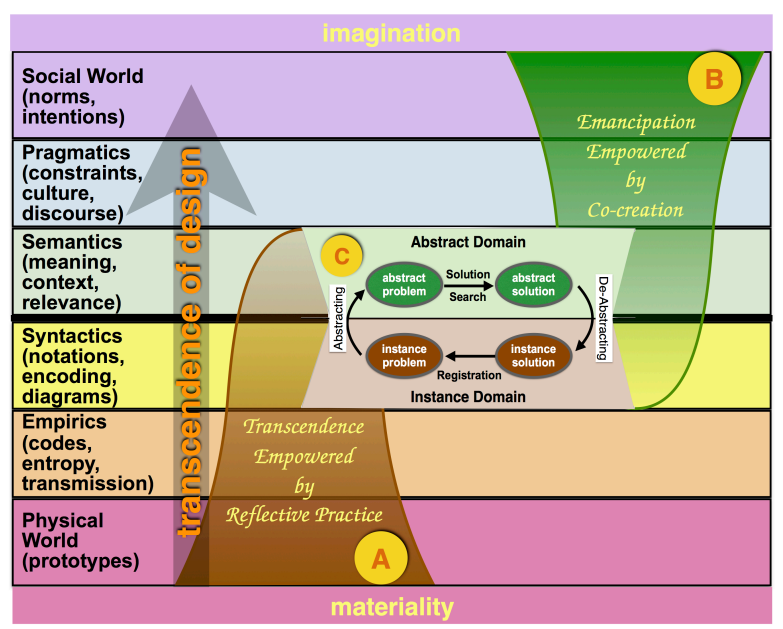

Figure 5. Realizing Intension in Design: Marrying Technical Rationality and Appreciative Systems

The Construction and medium of construction remain primarily grounded in technical rationality engaging objective, structural design properties (e.g. modularization, cohesion, encapsulation, composition of function, scale and stepwise refinement) [53]. These properties adhere to ontology engaging technological and computational resources of the semiotic framework: physical, empirics, and syntactics. The Design Intension and the Stakeholder Experience are primarily grounded in an operative appreciative system of psychology and sociology engaging subjective, aesthetic design properties (e.g. extensibility, patterns, reliability, transparency, programmability, identity, correctness, user friendliness, and elegance) [53]. These properties adhere to the ideation and co-creativity of the semiotic framework: semantic, pragmatic, and social world.

The Ontology of Structure \& Function articulates the artefact's stakeholder-facing interface as an ontological distillation of the Design Intension. As a metaphor of their intension, it invites each of the stakeholders to recognize their distinctive world-view in the artefact. In 
juxtaposition, the medium of construction lays out the means of materializing the artefact. Together they form the threshold of design (C) for the artefact at the core of the design process. (See Figure 5.) Throughout cycles of development and deployment, reflection (renewal and/or refactoring) incrementally recalibrates the ontology to attend to the inevitable evolution of: the composite appreciative system, the medium of construction technology, and the social context $[8,9,14,54]$.

\subsection{Taking the Cure}

Designer transcendence is a crucial ingredient in this marriage. The designers must reach beyond their traditional affinity for objective and technically rational measures of design quality to enfold a more sympathetic, subjective conception of quality that recognizes the immensely open system of the stakeholders' social context. The stakeholders in turn must accept the designers not only as craftspeople of the medium of construction but also, as full partners in the development and articulation of the Design Intension. Together their collaboration enriches the co-reflective practice of aligning intensions, appreciative systems, technology and the dynamic social context that form the challenging design spaces that characterize the $21^{\text {st }}$ century.

When imagination and materiality fuse in co-creative design, the artefact that results manifests effectiveness and efficiency. Technical rationality adroitly serves the objective nature of efficiency, but it is philosophically agnostic to the often-subjective nature of effectiveness. The psychosis in information systems design we need to cure is the belief that "doubling down" on technical rationality can somehow compensate for underestimating the necessity of appreciative systems.

\section{Conclusion}

We advocate for co-creative design practitioners, and information systems professionals specifically, as empowered and enfranchised agents capable of navigating between appreciative systems of all stakeholders, including the designer/developer [23, 35]. Particularly in conversation with malleable materials of design and construction, the epistemology of a reflective design practice that is also engaged in continuous experimentation and learning [18], provides a transcendent level of awareness necessary for effective co-creative design practice. Design actions and design outcomes represent the realm of the actual and empirical discussed in critical realism. To reflect and question governing mechanisms, and beliefs regarding them, leads to the transcendence possible in design practice with the perpetual inertia inherent in an epistemology of practice that is reflective in nature. There also is an imperative for transcendence in reflective practice where the discretion to select from (and act within) a spectrum of epistemic guises is a hallmark trait.

\section{References}

[1] Beck, K. (2004). Extreme Programming Explained: Embrace Change, 2ed. Addison-Wesley, Reading, MA.

[2] Bhattacherjee, A. (2001). "Understanding Information Systems Continuance: An Expectation-Confirmation Model." MIS Quarterly, 25(3), pp. 351-370.

[3] Checkland, P. \& Casar, A. (1986). “"Vickers' concept of an appreciative system: A systemic account." J. of Applied Systems Analysis, 13, pp. 109-115.

[4] Checkland, P., (1999). Systems Thinking, Systems Practice. John Wiley \& Sons, Chichester, UK.

[5] Checkland, P. \& Scholes, J. (1990). Soft Systems Methodology in Action. John Wiley \& Sons, Chichester, UK.

[6] Conboy, K., Gleasure, R. \& Cullina, E. (2015). "Agile Design Science Research," DESRIST 2015, B. Donnellan et al. (Eds.), LNCS 9073, pp. 168-180. Springer International.

[7] Dorst, K. \& Cross, N. (2001). "Creativity in the Design Process: Co-evolution of Problem-Solution." Design Studies, 22(5), pp. 425-437.

[8] Drechsler, A. \& Hevner, A. R. (2016). "A Four-cycle Model of IS Design Science Research: Capturing the Dynamic Nature of IS Artifact Design," DESRIST 2016, Eleventh International Conference on Design Science Research in Information Systems, pp. 1-8, St. John, Canada.

[9] Gill, T. G. \& Hevner, A. R. (2013). "A Fitness-Utility Model for Design Science Research." ACM Transactions on MIS, 4(2), 5 .

[10] Gregor, S. (2006). "The Nature of Theory in Information Systems." MIS Quarterly, 30(3), pp. 611-642.

[11] Gregor, S. \& Hevner, A. R. (2013). "Positioning and Presenting Design Science Research for Maximum Impact." MIS Quarterly, 37(2), pp. 337-355.

[12] Heeks, R. (2006). "Health information systems: Failure, success and improvisation." International J. of Medical Informatics, 75(2), pp. 125-137.

[13] Hevner, A. R. (2007). "A Three Cycle View of Design Science Research." Scandinavian J. of Information Systems, 19(2), pp. 87-92.

[14] Hirschheim, R. \& Klein, K, H. (1994). "Realizing emancipatory principles in information systems development: The case for ETHICS.” MIS Quarterly, 18(1), pp. 83-109.

[15] Hodgkinson, G. P. \& Starkey, K. (2012). "Extending the Foundations and Reach of Design Science: Further Reflections on the Role of Critical Realism." British J. of Management, 23(4), pp. 605-610.

[16] Hudson, J. R. https://johnrhudson.me.uk/systems /Appreciative_systems.pdf, accessed 11 April 2017.

[17] Iivari, J., Hirschheim, R. \& Klein, H. K. (2000). “A Dynamic Framework for Classifying Information Systems Development Methodologies and Approaches." J. of MIS, 17(3), pp. 179-218.

[18] Kim, G., Debois, P., Willis, J. \& Humble, J. (2016). The DevOps Handbook: How to Create World-Class Agility, Reliability, and Security in Technology Organizations. IT Revolution Press, Portland, OR. 
[19] Kohler, T., Fueller, J., Matzler, K. \& Stieger, D. (2011). "Cocreation in Virtual Worlds: The Design of the User Experience." MIS Quarterly, 35(3), pp. 773-788.

[20] Lakoff, G. \& Johnson, M. (1980). Metaphors We Live By. University of Chicago Press, Chicago, IL.

[21] Lakoff, G. \& Johnson, M. (1999). Philosophy in the Flesh. Basic Books, New York, NY.

[22] Lee, J. S., Pries-Heje, J. \& Baskerville, R. (2011). "Theorizing in Design Science Research," DESRIST 2011, Jain, H., Sinha, A. P. \& Vitharana, P. (Eds.), LNCS 6629, pp. 1-16. Springer-Verlag.

[23] Liu, K. (2000). Semiotics in Information Systems Engineering. Cambridge University Press, Cambridge, UK.

[24] Lyytinen, K. \& Hirschheim, R. (1987). "Information Systems Failures: A Survey and Classification of the Empirical Literature." Oxford Surveys in Information Technology, 4(1), pp. 257-309.

[25] Lyytinen, K. \& Robey, D. (1999). "Learning Failure in Information Systems Development." Information Systems Journal, 9(2), pp. 85-101.

[26] Markus, M. L. (1983). "Power, politics, and MIS implementation." Communications of the ACM, 26(6), pp. 430-444.

[27] Markus, M. L., Axline, S., Petrie, D. \& Tanis, S. C. (2000). "Learning from Adopters' Experiences with ERP: Problems Encountered and Success Achieved." J. of Information Technology, 15(4), pp. 245-265.

[28] McColl-Kennedy, J. R., Vargo, S. L., Dagger, T. S., Sweeney, J. \& Kasteren, Y. van. (2012). "Health Care Customer Value Cocreation Practice Styles." J. of Service Research, 15(4), pp. 370-389.

[29] McGilchrist, I. (2009). The Master and his Emissary. Yale University Press, New Haven, CT.

[30] Mills, C. W. (2000). The Sociological Imagination. Oxford University Press, New York, NY.

[31] Mingers, J. (2004). "Real-izing Information Systems: Critical Realism as an Underpinning Philosophy for Information Systems." Information and Organization, 14(2), pp. 87-103.

[32] Mumford, E. (2006). "The Story of Socio-technical Design: Reflections on its Successes, Failures and Potential." Information Systems Journal, 16(4), pp. 317-342.

[33] Ngwenyama, O. K. \& Lee, A. S. (1997). "Communication richness in electronic mail: Critical social theory and the contextuality of meaning." MIS Quarterly, 21(2), pp. 145-167. [34] Osborne, S. P., Radnor, Z. \& Strokosch, K. (2016). “CoProduction and the Co-Creation of Value in Public Services: A Suitable Case for Treatment?" Public Management Review, 18(5), pp. 639-653.

[35] Park, J. \& Park, H. A. (2014). “Co-creation Patterns between Designers and Users in the Design Process: A View of Reflexivity," DESRIST 2014, Tremblay, M. C. et al. (Eds.), LNCS 8463, pp. 32-53. Springer International.

[36] Peffers, K., Tuunanen, T., Rothenberger, M. \& Chatterjee, S. (2008). "A Design Science Research Methodology for Information Systems Research." J. of MIS, 24(3), pp. 45-77.

[37] Polanyi, M. (1969). Knowing and Being: Essays by Michael Polanyi. University of Chicago Press, Chicago, IL.

[38] Prahalad, C. K.; Ramaswamy, V. (2004). "Co-Creation Experiences: The Next Practice in Value Creation." J. of Interactive Marketing, 18(3), pp. 5-14.
[39] Ralph, P. \& Wand, Y. (2009). "A proposal for a formal definition of the design concept." In Lyytinen, K., Loucopoulos, P., Mylopoulos, J. \& Robinson, W., editors, Design Requirements Workshop (LNBIP 14), pp. 103-136. Springer-Verlag, London, UK.

[40] Rubin, K. S. (2012). Essential Scrum: A Practical Guide to the Most Popular Agile Process. Addison-Wesley, Reading, MA.

[41] Sanders, E. B.-N. \& Stappers, P. J. (2008). "Co-creation and the New Landscapes of Design." CoDesign, 4(1), pp. 518.

[42] Schön, D. A. (1963). Displacement of Concepts. Routledge, Abington, UK.

[43] Schön, D. A. (1971). Beyond the Stable State: Public and Private Learning in a Changing Society. Random House, New York, NY.

[44] Schön, D. A. (1986). Educating the Reflective Practitioner: Toward a New Design for Teaching and Learning in the Professions. Jossey-Bass, San Francisco, CA. [45] Schwaber, K. (2004). Agile Project Management with Scrum. Microsoft Press, Redmond, WA.

[46] Simon, H. (1996). The Sciences of the Artificial, 3ed. MIT Press, Cambridge, MA.

[47] Skyttner, L. (2005). General Systems Theory, 2ed. World Scientific Publishing Co., Singapore.

[48] Stamper, R. K. (1973). Information in Business and Administrative Systems. John Wiley \& Sons, New York, NY.

[49] Stamper, R. K., Althous, K. \& Backhouse, J. (1988). "MEASUR, Method for Eliciting, Analyzing, and Specifying User Requirements," In Olle, T. W., Verrijn-Stuart, A. A. \& Bhabuts, L., (Eds.), Computerized Assistance During the Information Life Cycle. Elsevier, Amsterdam, The Netherlands.

[50] Tesler, L., "The Law of Conservation of Complexity," http://www.designingforinteraction.com/tesler.html, retrieved 2 June 2017.

[51] Vickers, G, The Art of Judgement. Harper Collins, New York, NY, 1983.

[52] Vidgen, R. \& Wang, X. (2009). "Coevolving Systems and the Organization of Agile Software Development." Information Systems Research, 20(3), pp. 355-376.

[53] Waguespack, L. J. (2010). Thriving Systems Theory and Metaphor-Driven Modeling. Springer-Verlag, London, UK.

[54] Waguespack, L. J., Babb, J. S. \& Yates, D. J. (2017). "'Refactoring' Refactoring," in 50th Hawaii International Conference on Systems Sciences, Waikoloa Village, HI.

[55] Waguespack, L. J. \& Schiano, W. T. (2013). "Thriving Systems Theory: An Emergent Information Systems Design Theory," in 46th Hawaii International Conference on Systems Sciences, Maui, HI.

[56] Weick, K. (1995). "What Theory Is Not, Theorizing Is." Administrative Science Quarterly, 40(3), pp. 385-390.

[57] Wikipedia.org, "Rationality," https://en.wikipedia.org/ wiki/Rationality, retrieved 12 June 2017.

[58] Williams, K., Chatterjee, S. \& Rossi, M. (2008). "Design of Emerging Digital Services: A Taxonomy.” European J. of Information Systems, 17(5), pp. 505-517. 